\title{
IoT - An Exquisitely Adequate Technology to Reinforce the "City of Future - Smart City"
}

\author{
Monika Kohli ${ }^{1}$, Rohit Tiwari ${ }^{2}$ \\ Assistant Professor, Information Technology \& Computer Engineering Department, \\ K.J. Institute of Engineering \& Technology, Savli, Vadodara, Gujarat, India ${ }^{1,2}$
}

\begin{abstract}
IoT (Internet of Things) is considered to be the backbone for divergent electronic devices with which the Smart Cities are furnished with. And such smart cities are becoming smarter than ever by virtue of the flourishing growth in state-of-the-art automated and metering technologies. This research paper emphases upon catering allinclusive scrutiny over the Smart City conceptualization with its impetus and utilization. Furthermore, this review characterizes about the technologies participating in the construction of IoT infrastructure for a Smart City, with the notion of predominant characteristics and elements of the same. And last but not least, there is an elucidation of real-life incidents all over the world, as well as the major challenges to be confronted by IoT in present and coming future.
\end{abstract}

Keywords: IoT (Internet of Things), Smart City, Smart Home, Sensor Network, Security, Reliability

\section{INTRODUCTION}

The metropolitan city areas are experiencing a breakneck surge in the density of the population there, which in turn causing an urge for an increasing demand for infrastructure and amenities to fulfil the city inhabitant's essential needs. Since all the digital gadgets, like smartphones, actuators and sensors, are not only able to interconnect but also are capable of communicating with one another over the Internet; these are acting as one of the driving forces required for the implementation of the IoT. And apart for being a noteworthy escalation for such digital gadgets, these gadgets are driving a huge business potential for IoT too. Fundamentally, a typical IoT prototype is all about having a worldwide network infrastructure used to facilitate the communication between shrewd and autoconfiguring digital electronics gadgets. IoT, being an extensively disseminated assortment of real-life electronic gadgets having meagre processing efficiency and memory storing competence, enacts as the vital component for the improvement of the smart city and its infrastructures in the terms of effectiveness, protection and dependability. In this research paper, the IoT is being deliberated and reviewed as an exquisitely adequate technology supporting the reinforcement of smart cities all over the globe.

\section{IOT TECHNOLOGIES FOR SMART CITIES}

The IoT can be very well considered as a broadband network infrastructure having the Internet as its point of convergence and using typical communication protocols. The core impression of IoT talks all about having omnipresence of objects that can not only be assessed, understood, implied, but also can cause the environment transformation. So, it can be very well assumed that the development of communication technologies and various objects is responsible for putting IoT into action. There are so many things involved in the IoT belonging to different categories including mobile devices and various objects to be used in chores, cooperating together working towards a customary purpose. The significant aspect of the IoT always is and will be the impression, it has on the lifespan of its users. Out of so many technologies involved in the implementation IoT, some are discussed as follows.

\section{A. Radio-Frequency Identification (RFID)}

RFID (Radio-Frequency Identification) is considered to be one of the significant technologies having a considerable contribution in the advancement and implementation of concepts like IoT. RFID, which is actually an amalgamation of working of tags and readers, not only providing a way to automatically recognize the electronic gadgets, which are part of the IoT network infrastructure, but also every such gadget in the network is dispensed with an inimitable digital identification. This RFID technology is required for all these electronic gadgets, so that these can remain not only assimilated into the global network but also interrelated to the digital information and amenities.

B. Wireless Sensor Network (WSN)

This Wireless Sensor Network (WSN) technological component of IoT is responsible for supplying diverse appropriate data. Apart from its (WSN's) capability to be integrated with RFID, to have an added advantage of procurement of 
crucial statistics viz. concerning the displacement, temperature, geographical location, WSN is a great aid in technical aspects in the smart services including smart health management wellness programs, ecological and government amenities.

\section{C. $\quad$ Addressing}

IoT can be compared with the Internet in terms of interconnection. While the Internet is making people to interconnect with each other, the IoT, to create smart milieus, is providing interconnection between electronic gadgets and appliances or anything which is capable enough to connect to a network. But if IoT has to work perfectly in the sense of efficiency and reliability, it is quite vital to be capable enough to uniquely identify the participating gadgets. And it is fairly imperative to provide unique addressing to this gigantic assortment of interconnected gadgets, as all these gadgets are going to be controlled through the Internet. The IoT must have an inimitable addressing mechanism fulfilling the substantial prerequisites, apart from the aforementioned notion of inimitability, along with perseverance, trustworthiness and extensibility.

\section{Middleware}

The Middleware performs a crucial part into the interconnecting of all participating gadgets with the core of the application layer. The reason being the gadgets having limited process and storage capabilities and there are quite a lot of issues faced not only because of the heterogeneous nature of these participating gadgets, but also the possible set of applications of IoT has immensely assorted applications. Essentially, the middleware is accountable for succinctly assimilating the functionalities and communication proficiencies of every single participating gadget.

\section{IOT APPLICATIONS FOR SMART CITIES}

Electronic gadgets and appliances, assorted in nature, are interconnected with one another by IoT by means of the Internet. By saying this, we are putting a point forward that for enhanced and effective accessibility in IoT, each and every participating gadgets and appliance should be connected to the Internet. There can also be the implementation and adequate use of sensors at the diversified geographical site, which will help to enhance the applicability by accumulating and scrutinizing the data. While the Preeminent applications of the IoT for smart cities are depicted in the Fig. 1, there are some actual smart city applications are deliberated below the Fig. 1.

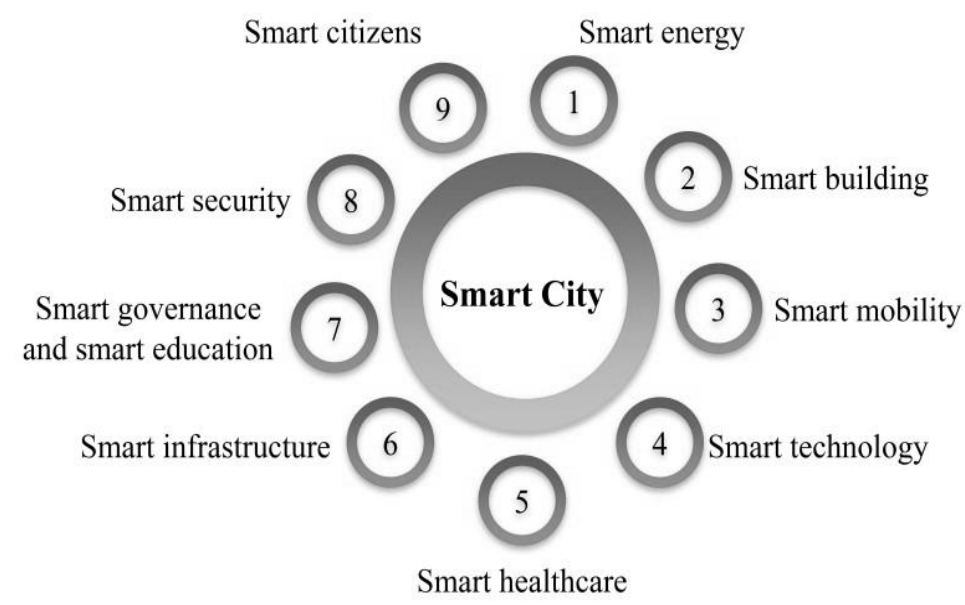

Fig. 1 IoT Application in a Smart City

\section{A. Smart Homes}

There will be sensors, in smart homes, generating data which will help to keep an eye over these homes. Such sensory data can be used to alert the residents of the smart homes about any unhealthy amendments in the environment e.g. level of pollution exceeding its borderline, be it sound, air or water pollution.

\section{B. Smart Parking Areas}

A smart city, with functioning smart parking areas disseminated in the city, will be able to track the arrival and departure of numerous automobiles in such parking areas. The architectural design of such car parking areas should be implemented accordingly by considering the number of cars in a particular region and these new smart parking areas ought to be created where there is larger accessibility of automobiles. Furthermore, such vital information gathered at these smart parking spaces can be used in a beneficial manner for the betterment of the residents of that particular smart city. 


\title{
International Journal of Advanced Research in Computer and Communication Engineering
}

\author{
Vol. 7, Issue 12, December 2018
}

C. Smart Weather and Water Systems

The contribution can be made to upsurge the effectiveness of the smart cities by implementing smart weather and water arrangement practices. In such arrangements, sensors will be used to accumulate diversified information about environmental elements including pressure, rainfall, temperature, air velocity, etc.

\section{Automobile Traffic}

Automobile Traffic in a city has a lot of useful vital data. In a smart city, this automobile traffic data can be collected intelligently and then with appropriate scrutiny, the generated information can be used for the betterment of both the administration and inhabitants. For example, one of such information can be used by the residents to determine the travel time to a location in the city.

E. Smart Environmental Pollution Management

Environmental Pollution is considered to as one of the biggest health hazards to the residents of a city. A smart city must have a smart environmental pollution management system which is, not only going to watch over the environmental pollution statistics but also will process the data and spread this useful information it's all inhabitants, particularly to the individuals having medical conditions. Furthermore, the data will be collected covering all pollution types viz. air, water and noise.

\section{F. Surveillance Systems}

From the point of view of the inhabitants of a smart city, their safety and security are a major aspect of life. Although, it's quite a challenging assignment to investigate the sensory data and to sensing criminal activities, yet the smart city must be under vigilance through effective and reliable surveillance systems.

\section{CHALLENGES FOR IOT IN THE SMART CITY}

The journey to create a perfect smart city is not a cakewalk. Even after a great number of technological advancements in the field of IoT, still there remain some major challenges as deliberated in this particular segment.

\section{A. Security and Privacy}

A system working copiously over the internet cannot guarantee a comprehensive safety, security and privacy. Same is the case with IoT enabled smart cities. As IoT is using a mutual platform to accumulate and scrutinize all the sensory data, it is not only getting susceptible to severe vulnerabilities, but also such systems are prone to quite a lot of intrusion activities. To make it worse, the leakage of data is a definite possibility due to all such security threats.

\section{B. Heterogeneity}

For the reason that the IoT serves to a greater variety of applications in the real world, its evolution has made its working infrastructure an amalgamation of heterogeneous gadgets and appliances with their individual exclusive usefulness in the context of a specific application. Now the biggest challenge in front of IoT system implementation team is that of the selection of adequate software and hardware solution and then establishing an appropriate network infrastructure with an acceptable cooperating arrangement, required to assimilate these participating heterogeneous gadgets and appliances.

\section{Trustworthiness}

The smart city, being an IoT enabled arrangement, is prone to reliability concerns. Almost all of the reliability apprehensions of IoT will be there to cause a problem. One instance can be, where a car's movement can make the communication unreliable. The situation is actually pretty worse as IoT is largely dependent on the participating smart gadgets and appliances, and their failure can damage the reliability aspect of the IoT enabled smart city.

\section{Large Scale of Implementation}

There can be some particular circumstances where the communication is taking place among an enormous number of participating gadgets disseminated over distant geographical locations. Although IoT systems are equipped with appropriate infrastructure to accumulate and scrutinize the data being received from dissimilar gadgets and appliances, it becomes quite tough to perform the same job over a larger scale. This is a big challenge as such a massive amount of data will require appropriate storing and processing proficiencies at a competitive increased rate. To make it worse, we have a geographically distant distribution of all participating gadgets obstructing their monitoring.

\section{E. Legal and Social Aspects}

The legal and social aspects of the IoT enabled smart cities cannot be overlooked, as the complete system is following a service model built and working upon the data provided by the end-user. It must be taken care of that all different 


\title{
International Journal of Advanced Research in Computer and Communication Engineering
}

\author{
Vol. 7, Issue 12, December 2018
}

international and local regulations and commandments are being practised by all the service providers. All end-users must be made aware of all working protocols through creative and innovative ways to maintain this law and order situation in control in the smart city.

F. Big Data

As the number of participating gadgets, sensors and appliances in the IoT system could cross a mark of fifty billion, there will be a massive volume of data being transported, stockpiled and scrutinized. And it is needless to say that such IoT infrastructure will be considered as some of the crucial reserves of big data.

\section{CONCLUSION}

A smart city is unquestionably going to become the city of future, having immense possibilities to represent the human life at its technically enriched form. In this research paper, the notion of IoT, being the most adequate technology for a good build establishment of the smart city, is deliberated. The participating component technologies have been discussed and then the applications of the IoT in the context of the smart city were investigated. Furthermore, the challenges going to be faced by the IoT system during the implementation of a smart city are being scrutinized. In a smart city, the IoT systems with all its capabilities and characteristics are very much able to preserve the privileges of the inhabitants of the smart city by employing sensory devices and intellectual systems.

\section{ACKNOWLEDGEMENT}

This work was supported by Dr. Ashok Kumar Jetawat, the authors thank to, for his kind guidance in the research and as reviewer to this research paper. The author would also thank the KJIT management for their astonishing support in the research.

\section{REFERENCES}

[1]. M. M. Rathore, A. Ahmad, A. Paul, and S. Rho, "Urban planning and building smart cities based on the internet of things using big data analytics", Comput. Netw., 2016, DOI: 10.1016/j.comnet.2015.12.023.

[2]. J. Gubbi, R. Buyya, S. Marusic, and M. Palaniswami, "Internet of Things (IoT): A vision, architectural elements, and future directions," Future Gener. Comput. Syst., vol. 29, pp. 1645-1660, 2013.

[3]. M. Shafie-khah, E. Heydarian-Forushani, M.E.H. Golshan, P. Siano, M.P. Moghaddam, M.K. Sheikh-El-Eslami, and J.P.S. Catalão, “Optimal trading of plug-in electric vehicle aggregation agents in a market environment for sustainability,” Appl. Energy, Volume 162, 15 January 2016, Pages 601-612.

[4]. N. Bressan, L. Bazzaco, N. Bui, P. Casari, L. Vangelista and M. Zorzi. "The deployment of a Smart Monitoring System using Wireless Sensor and Actuator Networks." Proc. of IEEE SmartGridComm 2010, Gaithersburg, MD, 2010.

[5]. H. Schaffers, N. Komninos, M. Pallot, B. Trousse, M. Nilsson, and A. Oliveira, "Smart Cities and the Future Internet: Towards Cooperation Frameworks for Open Innovation", The Future Internet, Lecture Notes in Computer Science Volume 6656, pp. 431-446, 2011.

[6]. J. P. Lynch, and J. L. Kenneth, "A summary review of wireless sensors and sensor networks for structural health monitoring." Shock and Vibration Digest vol. 38 no. 2, pp. 91-130, 2006.

[7]. X. Li, W. Shu, M. Li, H.-Y. Huang, P.-E. Luo, and M.-Y. Wu, "Performance evaluation of vehicle-based mobile sensor networks for traffic monitoring." IEEE Transactions on Vehicular Technology, vol. 58, no. 4, pp. 1647-1653, May 2009.

[8]. N. Bui and M. Zorzi. "Health Care applications: A solution based on the Internet of Things." In ISABEL, Barcelona, Spain, Oct. 2011.

[9]. R. Bonetto, N. Bui, V. Lakkundi, A. Olivereau, A. Serbanati and M. Rossi. "Secure Communication for Smart IoT Objects: Protocol Stacks, Use Cases and Practical Examples." IEEE IoT-SoS, 2012, San Francisco, CA, US.

[10]. Association Institutes Carnot White Paper, -Smart Sensored Objects and Internet of Things, $\|$ Greece, 2011.

[11]. Alamri, A.; Ansari,W.S.; Hassan, M.M.; Hossain, M.S.; Alelaiwi, A.; Hossain, M.A. A Survey on Sensor-Cloud: Architecture, Applications, And Approaches. Int. J. Distrib. Sens. Netw. 2013, 9, 917923.

[12]. Elmangoush, A.; Alhazmi, A.; Magedanz, T.; Schuch, W.; Estevez, C.; Ehijo, A.; Wu, J.; Et Al. Towards Unified Smart City Communication Platforms. In Proceedings of The Workshop on Research in Information Systems and Technologies, Chillán, Chile, 16 October 2015.

[13]. A. Gluhak, S. Krco, M. Nati, D. Pfisterer, N. Mitton, and T. Razafindralambo, "A Survey on Facilities for Experimental Internet of Things Research," Communications Magazine, IEEE, vol. 49, no. 11, pp. 58-67, 2011.

[14]. S. Sicaria, A. Rizzardia, L. Griecob, and A. Coen-Porisinia, "Security, Privacy and Trust in Internet of Things: The Road Ahead," Computer Networks, vol. 76, pp. $146-164,2015$

[15]. I. Vilajosana, J. Llosa, B. Martinez, M. Domingo-Prieto, A. Angles, and X. Vilajosana, "Bootstrapping Smart Cities Through a Self-sustainable Model based on Big Data Flows," Communications Magazine, IEEE, vol. 51, no. 6, pp. 128-134, 2013. 